RHIC-PG-1

\title{
INTRABEAM SCATTERING COMPUTER CALCULATIONS AND \\ OTHER PERFORMANCE ISSUES
}

\author{
A.G. Ruggiero
}

(BNL, November 17, 1983) 


$$
A G R ; \text { nov. } 17,1983
$$

(1)

Intrabeam Scattering Computer Calculations

Smooth Lattice $(\eta=\beta=0)$

$$
\beta=30 \mathrm{~m} \quad \eta=20 \mathrm{~m}
$$

$\delta$, rms relive energy spread

$\varepsilon=2 \pi \sigma^{2} / \beta$, rms betatron imittance

$$
\begin{aligned}
& \tau_{\beta}^{-1}=\dot{\varepsilon} / 2 \varepsilon \\
& \tau_{E-1}^{-1}=\dot{\delta} / \delta
\end{aligned}
$$

$$
\left(\tau_{E}^{-1}\right)_{\text {Bunted }}=0.5 \times\left(\tau_{i}^{-1}\right)_{\text {unamended }}
$$

I, average current for unibumaded beanie = Ne f trevor. , peak invent for buanadid deans For bumped beam

$\beta c$ : bean: velocity

$N$, no If parties in the bunch

- Oe, rms: bunch length

Case considered: Gold (Avi) $A=197 \quad Z=79$

The numbers in to next two tables are for

$$
I=1 m A \text {-particle }
$$

Emitionas given: ace normalized

$\tau_{\beta}$ is for horizontal plane. The vertical enrittance is stable or slightly damped: 
$\varepsilon_{H} \varepsilon_{V} \varepsilon: \tau_{E}^{-1}: \tau_{\beta}^{-1}$

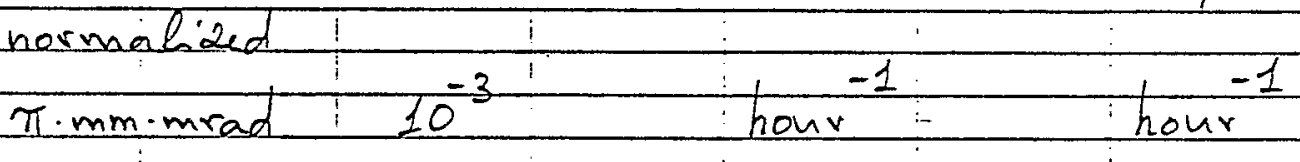

case

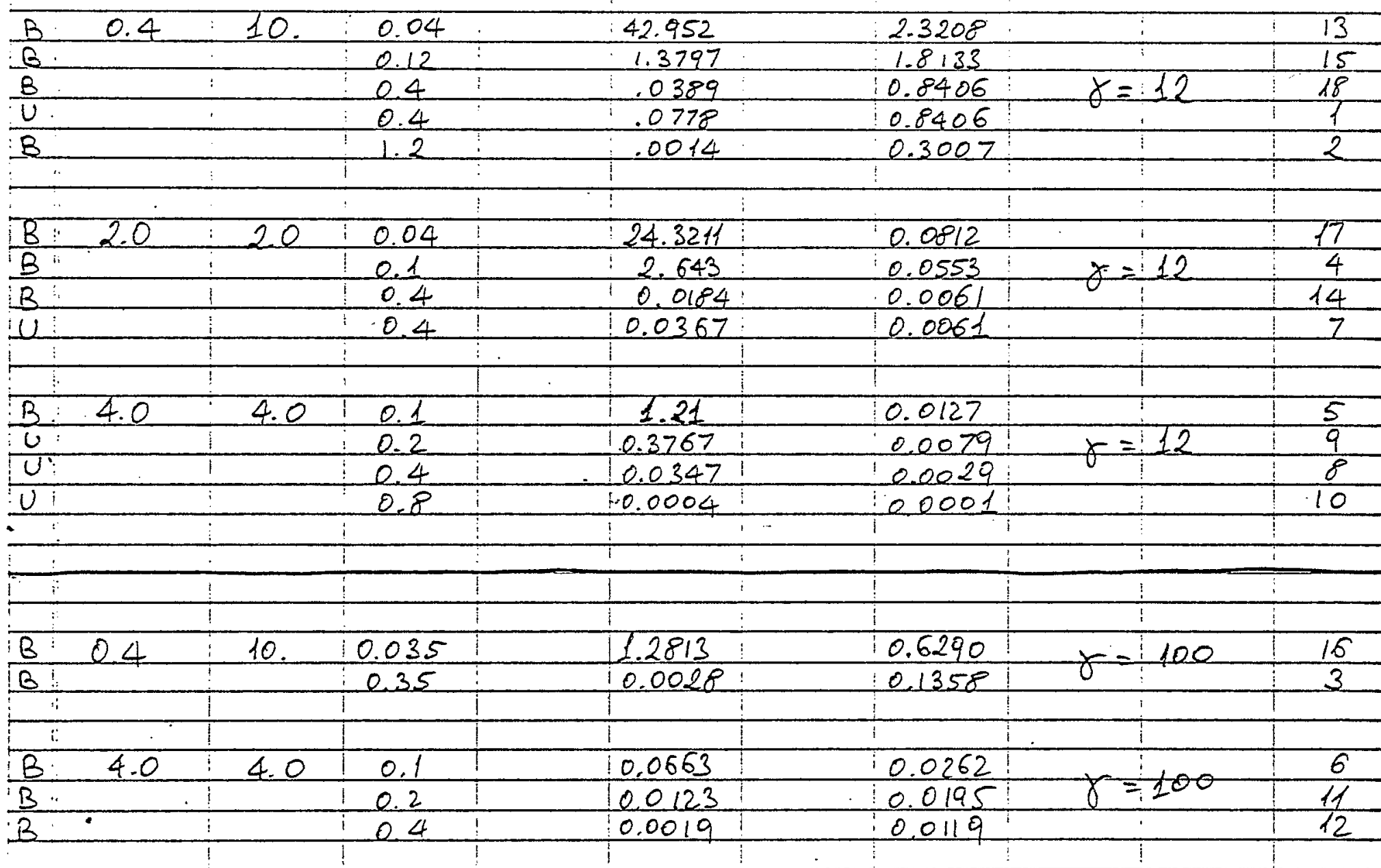

Eightieen $\left(\frac{18}{1}\right)$ cates have been cohridered

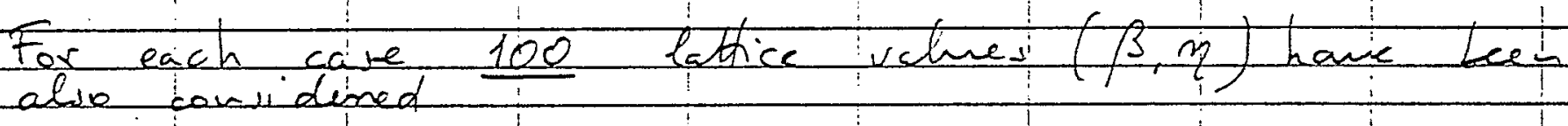

abse convidined
$\left\{\begin{array}{l}\beta=5 \text { to } 50, \text { step } 5 \text { imctar } \\ \eta=0.5 \text { to } 5 ., \text { step } 0.5 \text { meters }\end{array}\right.$ 
(3.)

Space Charge Calanlation At Injection in the Boorter

$E_{N}$, normalized ermittance

Actual emittance, $\varepsilon=6 \pi \sigma^{2} / \beta_{\text {tat }}=\varepsilon_{N} /(\beta \gamma)$

$N_{B}$, total number of panticter injected into the Boortex Space coarge Limit formula is

$$
\frac{N_{B}}{\varepsilon_{N}}=\left(\beta \gamma^{2}\right) \frac{\pi B_{g} \Delta D}{2 r_{0} F} \frac{A}{Q_{i}^{2}}
$$

Ascumption: "rownd" beann; that is i $\varepsilon_{H}=E_{V}$

Be, buncain factor $=0: 5$

$\Delta v$, betetion-tune Luprizusion $=0.1$

$r_{0}=1,535 \times 10^{18} \mathrm{~m}$, classical proton radins

$A$, atomic mais $=1997$ jor Gold

$Q$ F charge statuis $=34$ partioly strifed iour

$F$, form jector $\sim 1$ for $\beta \ll 1$ and $\gamma \sim 1$

We take a Booster Aceptance of $40 \pi \mathrm{mm}$-mrad and we cscume we ditho the the feam, by steaning lanch multi-tirn injection, unite tồ's ajerture is fuel and the shace charge limat is rieached

Te following fatte ixplores the effect of the in irecti Kinctic emergy. (T) on the sface charge timit 
(4)

Procedure.

I: Assign Kivetic injection energy, $T$

is Calanhale relchivishic Jaches $\beta$ and $\gamma$

IIL. Calcalahe ptare-space hendity at the himit $N_{B} / \varepsilon_{N}$; frow (I)

iv. Calarlate Normaliaed Emittance from

$$
\varepsilon_{N}=(\beta \gamma)(40 \pi \mathrm{mm}: \mathrm{mrad})
$$

$\checkmark$ Caloulate nusater of pantictes $N_{B}$ that can be injected int Le. Boartex

vi Assume 3 bunches tand a 50\% efficiengy for fund: striping Letwreen Booster and AGs

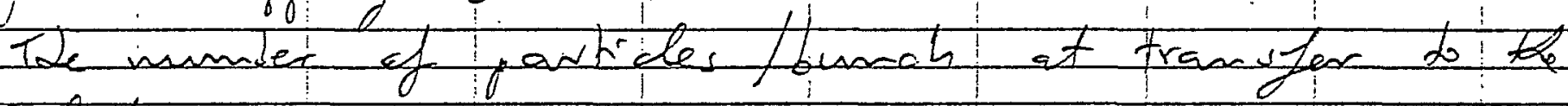
Collider is:

$$
N=N_{B} /(2 \times 3)
$$

Vir: Clantate cwohtion peniod $\tau: C</ \beta c$

$C$, Beoster ciranguerence $=201.75 \mathrm{~m}$

Viii Assume a pulie prom th tandern 250 pree and ia d.c cuvirent of $4.4 \mu$ Amp - piartide!

Calculate no. of pastide, per: turn

$$
N_{B} / \tan =(4.4 \text { pitmp-ipant }) \times \tau
$$

ix: Calontate requined unambere of turine at injection to get at the tobl $N_{B}$

$$
\text { no of turns }=N N_{B} /\left(N / t_{\text {irn }}\right)
$$

x: Celonkte reguined: pulse bength $=($ no. of turns $) \times \tau$ 


\begin{tabular}{|c|c|c|c|c|c|c|c|}
\hline$T$ & $\beta$ & 8 & & $N_{g} / \varepsilon_{N}$ & $\varepsilon_{N}$ & & $N_{B}$ \\
\hline $\mathrm{HeV}$ & & & & $\pi^{-1-1-1}$ & $\pi$ mm:mada & & \\
\hline & .04613 & .001066 & & $4 \times 10^{14}$ & 1.85 & & $7.4 \times 10^{8}$ \\
\hline 1.5 & .05648 & .001599 & & $5 \times 10^{14}$ & 2.26 & & $1.13 \times 10^{9}$ \\
\hline 2. & .06519 & .002132 & & $5.7 \times 10^{14}$ & 2.61 & & $1.5 \times 10^{9}$ \\
\hline 3. & .07978 & .003197 & & $7 \times 10^{14}$ & 3.20 & & $2.24 \times 10^{9}$ \\
\hline 4. & .09204 & 004263 & & $81 \times 10^{14}$ & 3.70 & & $3 \times 10^{2}$ \\
\hline 5 & .10283 & 005329 & & $9 / \times 10^{4}$ & 4.115 & & $3.74 \times 10^{9}$ \\
\hline- & & & & & & & \\
\hline & & & & & & & \\
\hline$T$ & $\tau$ & & $N$ & $N_{s} /$ turn & & nno. Thin & 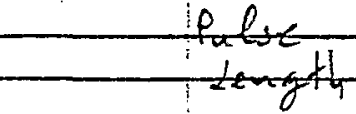 \\
\hline $\mathrm{MeV}$ & $\mu \mathrm{sec}$ & & $\times 10^{8}$ & $\times 10^{8}$ & & & $\theta$ \\
\hline & & & 11 & 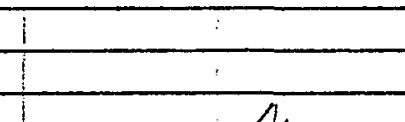 & & 180 & 2 \\
\hline$\frac{1 .}{1.5}$ & $\frac{14.6}{11.9}$ & & 1.88 & $\begin{array}{l}4.3 \\
3.3\end{array}$ & & 3.4 & 40 \\
\hline 2. & 10.3 & & 248 & 2.83 & & 5.3 & 54. \\
\hline 3. & 8.43 & & 3.73 & 2.32 & & 9.7 & $8 \%$ \\
\hline 4. & 7.3 & & 5.0 & 20 & & 15 & $10 \%$ \\
\hline 5. & 6.54 & & 6.24 & 18 & & 21 & 13 \\
\hline & & & & & & & \\
\hline & & & & & & & \\
\hline & & & & & & & \\
\hline & & & & & & & $E$ \\
\hline
\end{tabular}


Performance

Long Bumcder, High Intersity

Grosing an Angle

$$
L=N_{c}^{2} B f_{\text {rew }} / 4 \pi\left(\frac{\alpha}{2} \sigma_{e}\right) \sigma_{v}
$$

$B$, no of bunceles $=3$

$N_{c}$, no of pastide /Bumah in collibing mode $=6 \times 10$

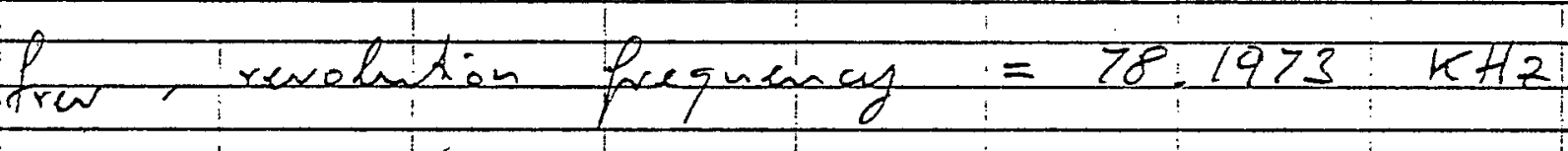
$\alpha$, toml, vrossing angle $=4 \mathrm{mrad}$ $\sigma_{e}$, ims banch length $=50 \mathrm{~m}$ $\sigma_{v}$, rme bram heright $=0.037 \mathrm{~mm}$ 
Longitudinal stability of the Loug Buncder $\left|\frac{Z}{n}\right|=\frac{E_{E}|\eta|}{e I P}\left(2 \frac{\sigma_{E}}{E}\right)^{2} \frac{A}{Z^{2}}$

$I_{p, \text { peak current }}=N_{c} \beta_{c} /\left(\theta_{e} \sqrt{2 \pi}\right)$

$N_{0,}$ we of pantide / branch $=6 \times 10^{60}$

$\sigma_{c}$, rms bunch langth $=50 \mathrm{~m} \quad\left(\sigma_{t}=0.17 \mu \mathrm{sec}\right)$

$I_{p}=0.023$ Amp-pankick $(\beta \sim 1)$

For Gold $\left(A_{u}\right) \quad A=191$ and $Z=19$

Hssume a couphing ingectance of $|z / \mathrm{h}|=10 \mathrm{imm}$

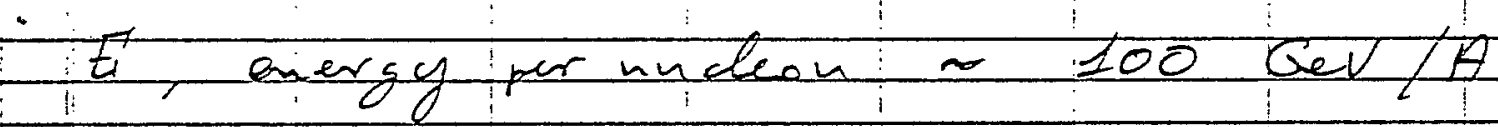

$\eta=\gamma_{T}^{2}-\gamma_{T}^{2} \quad, \gamma_{T}$ tramition energy /rect exengy

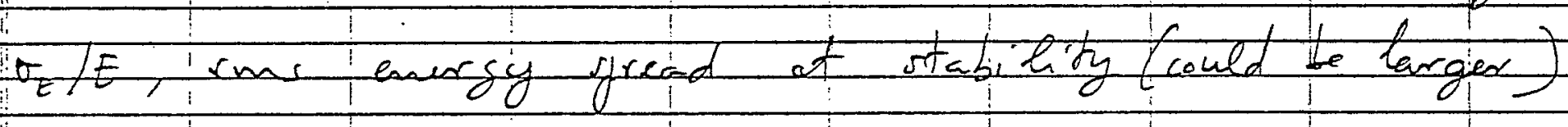
$B$, bunch arca $=6 \pi \sigma_{E} \cdot \sigma_{\tau}$

Assuming 3 bumad fer tean the thal ares If each beam is

\section{8}

The jelowing talle explones to dyendence with $\gamma_{T}$ of the thicumbold spread $\sigma_{E} / E$ at $100 \mathrm{GeV} / \mathrm{A}$ and

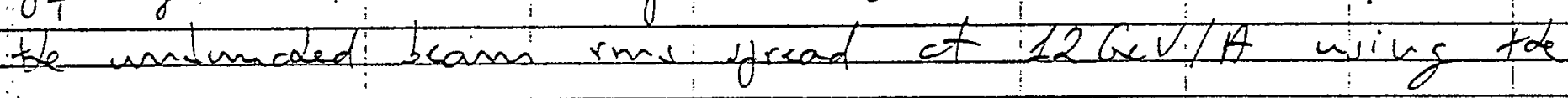
frmuka $\quad 3 B=4 T_{\text {rwol }} \sigma_{E}, \quad I_{\text {rw }}=12.788 \mu \mathrm{\mu kc}$ 


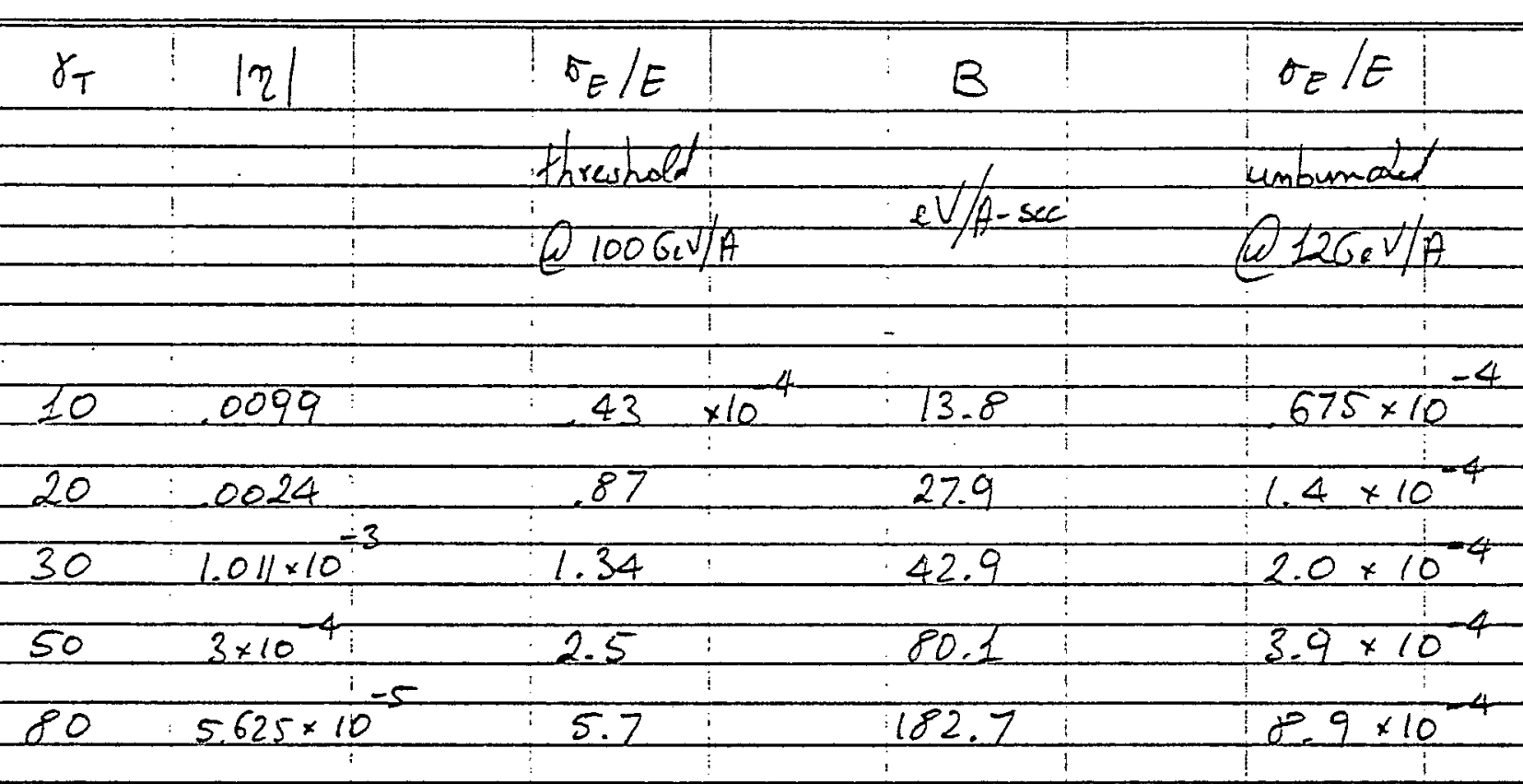

In the following table $T$ is the kivetic enorgy at

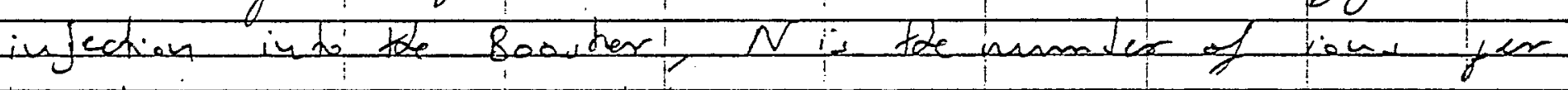
Lunch at transfer Itfureen AGS and Collider

We ausune it the are 3 Lunder' in the boouter, ouly pere 3 bunster ane accelexathed in the AGS at

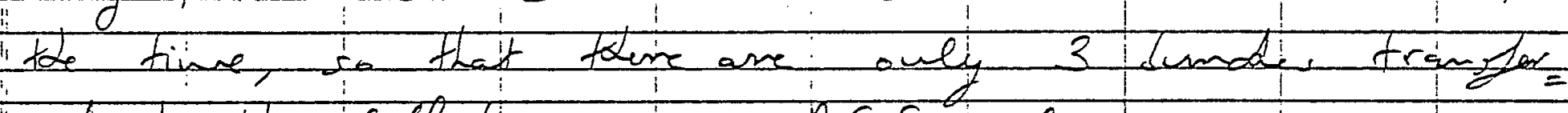
ned to the collidir verry as ipulse

$n$, nomber of bumcher from the $A G S$ requived to get $3 \times 6 \times 10^{10}=18 \times 10^{4}$ ion fer bieans

The mumber of AGS julke ame the fore $n / 3$

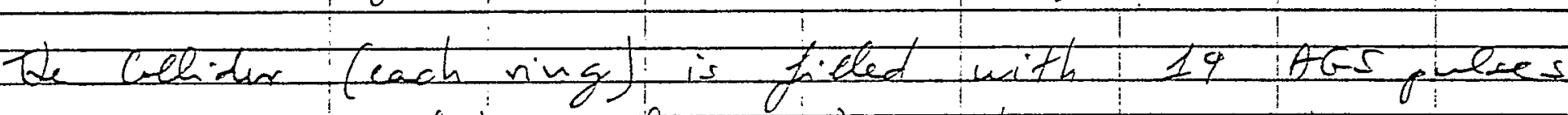
in a box-car' fouhion $(h=57)$ and $n$; RF ickadking toles

Te jilhing hive is calarked ussuming two mi muts for a $R F$ stacking cujde $=(1$ min $A G S+1 \mathrm{~min} R F)$ 


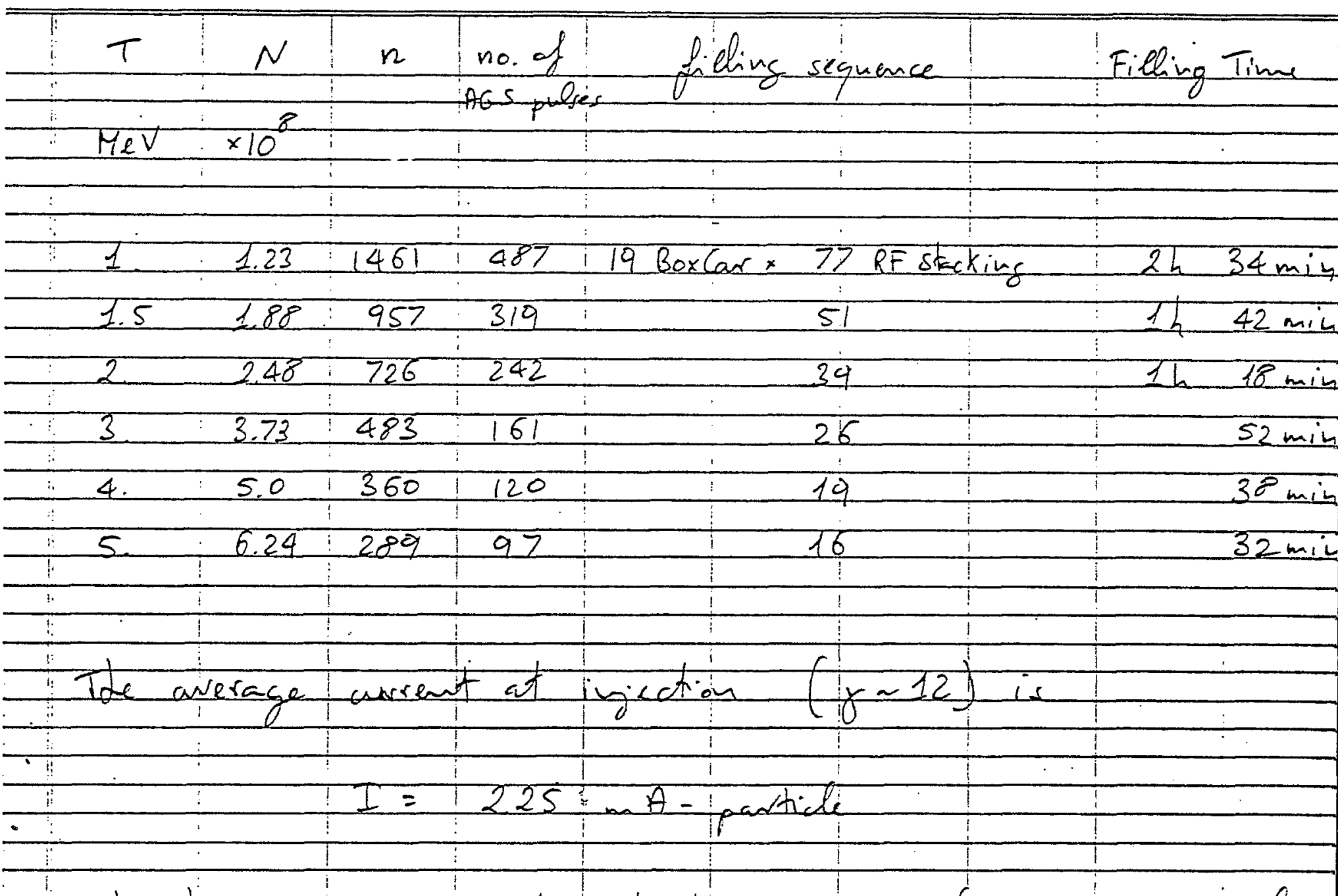

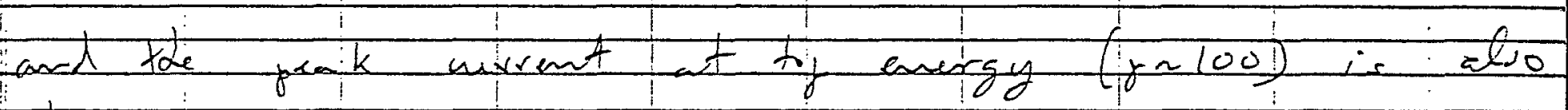
to sane 
Take $\quad Y_{T}=10$
$\sigma_{E} / E$
(a) $100 \mathrm{GeV} / \mathrm{A}$
$.43 \times 10^{-4}$
$\sigma=/ E @ 12 G \mathrm{G} / \mathrm{A}$
$.675 \times 10^{4}$
$\varepsilon_{N}$
$\gamma$
$t_{E}$
$t_{\beta}$

$\pi \cdot \operatorname{mm} \cdot \operatorname{mrad}$

2

4.

12

12

0.03

0.09

100.

4.

100 hours

hours

$\begin{array}{l:l}2 & 100 . \\ 4 . & 100 .\end{array}$

6.

20.

Individud Bumch Area of trangufer from the AGS to toe collider

$$
S=0.014 \mathrm{iV} / \mathrm{A}-\mathrm{sec} / 0.072
$$

having inchided a $50 \%$ dibution factor far tranjjer, RF stacking and Accelerativ. 


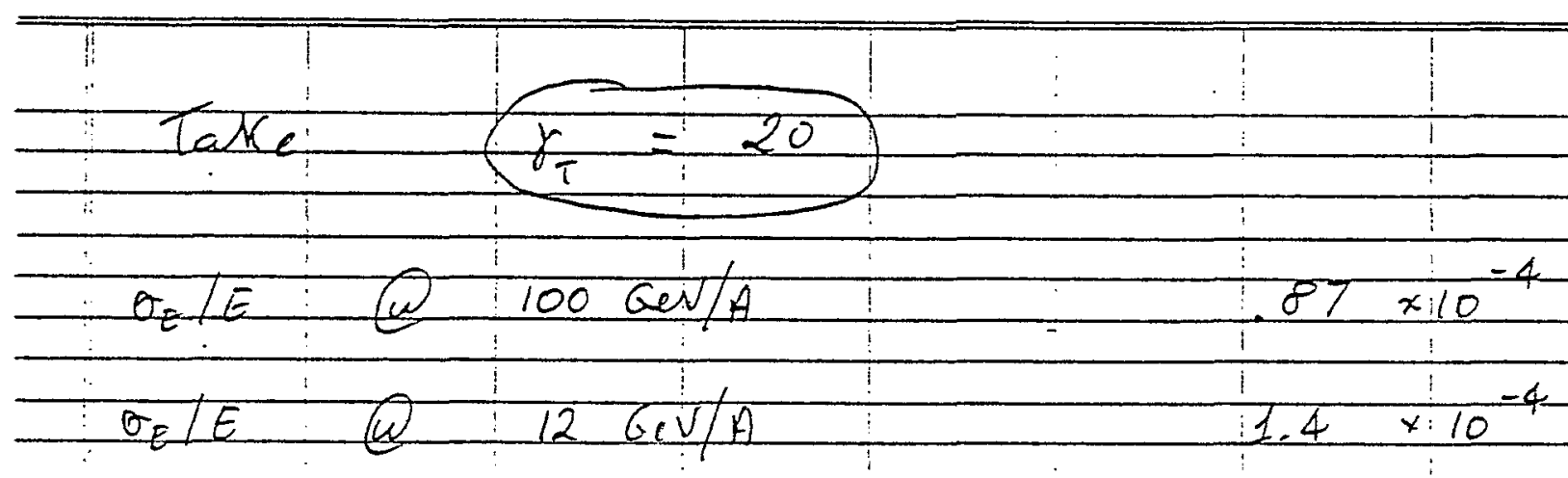

$\varepsilon_{N}-\gamma$

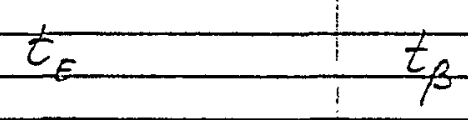

$\pi \cdot \operatorname{mm} \cdot \operatorname{mrad}$

hones: hours

\begin{tabular}{l|l|l}
2 & 12 \\
\hline & & 12
\end{tabular}

0.12

4.

12.

0.3

9

40 .

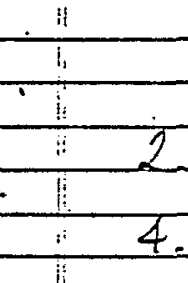

\begin{tabular}{l}
12. \\
\hline 1 \\
1100 \\
100 \\
1
\end{tabular}

Indinidrad Bundu Ariea ot transfer frow the ACS to cothidex

$$
S_{1}=0.024 \mathrm{~V} / \mathrm{A}-\mathrm{sec}
$$

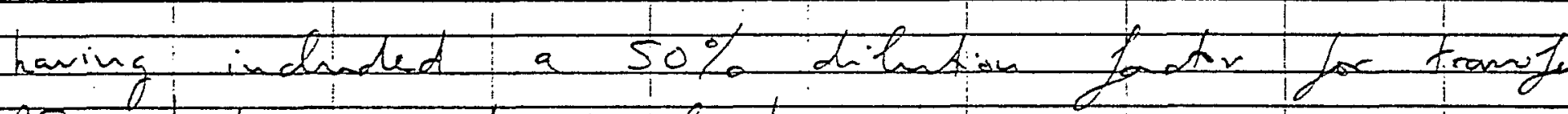
RE stacking and Acculeration, 


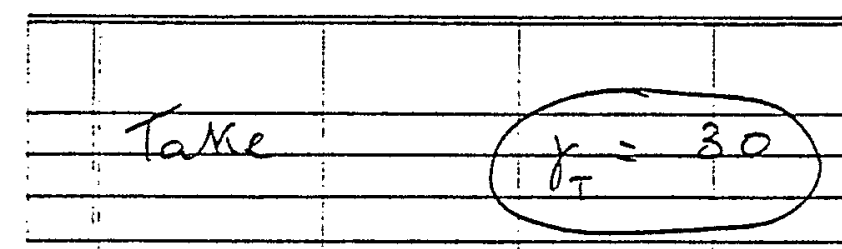
$\sigma_{E} / E$
(2) $100 \mathrm{Gev} / \mathrm{A}$
$1.34 \times 10^{-4}$
$\sigma_{E} / E$
$\omega: 12 \mathrm{GeV} / \mathrm{A}$
$20 \times 10^{-4}$

$\varepsilon_{N}$

$\pi$ mm-mrod

\begin{tabular}{l|l}
2 & 1 \\
\hline &
\end{tabular}

4.

2

4. $\gamma$

\begin{tabular}{c|c|c} 
& & hours \\
\hline 12. & & 0.5 \\
\hline 12 & & 1.2 \\
& & \\
\hline 100 & & - \\
\hline 100 & & 0
\end{tabular}

$t_{\beta}$

dours

25

56

20.

Indivitural Bunch Area at Tramejer from to AGs th te collden

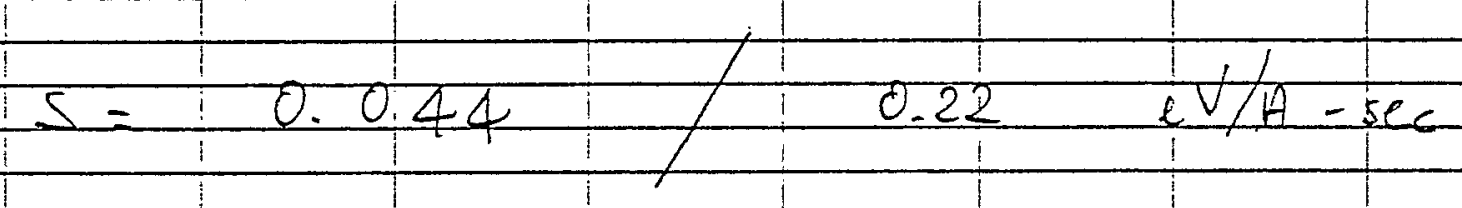

having indioded a $50 \%$ duturion jactor for trancyer, RE stacking and Hecelerntor.

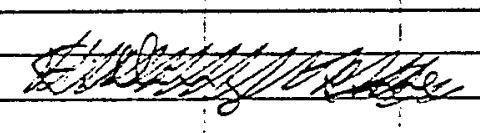




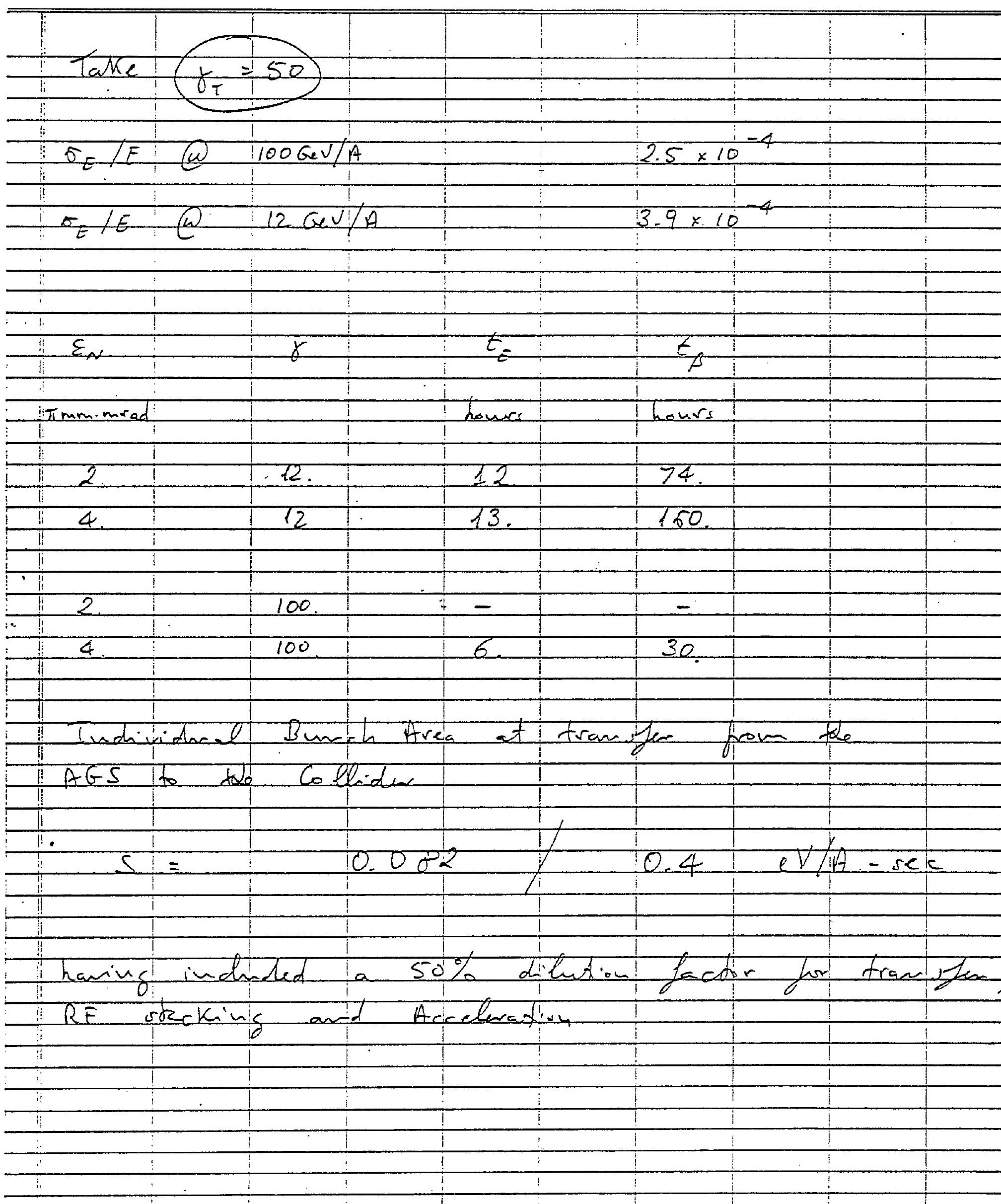



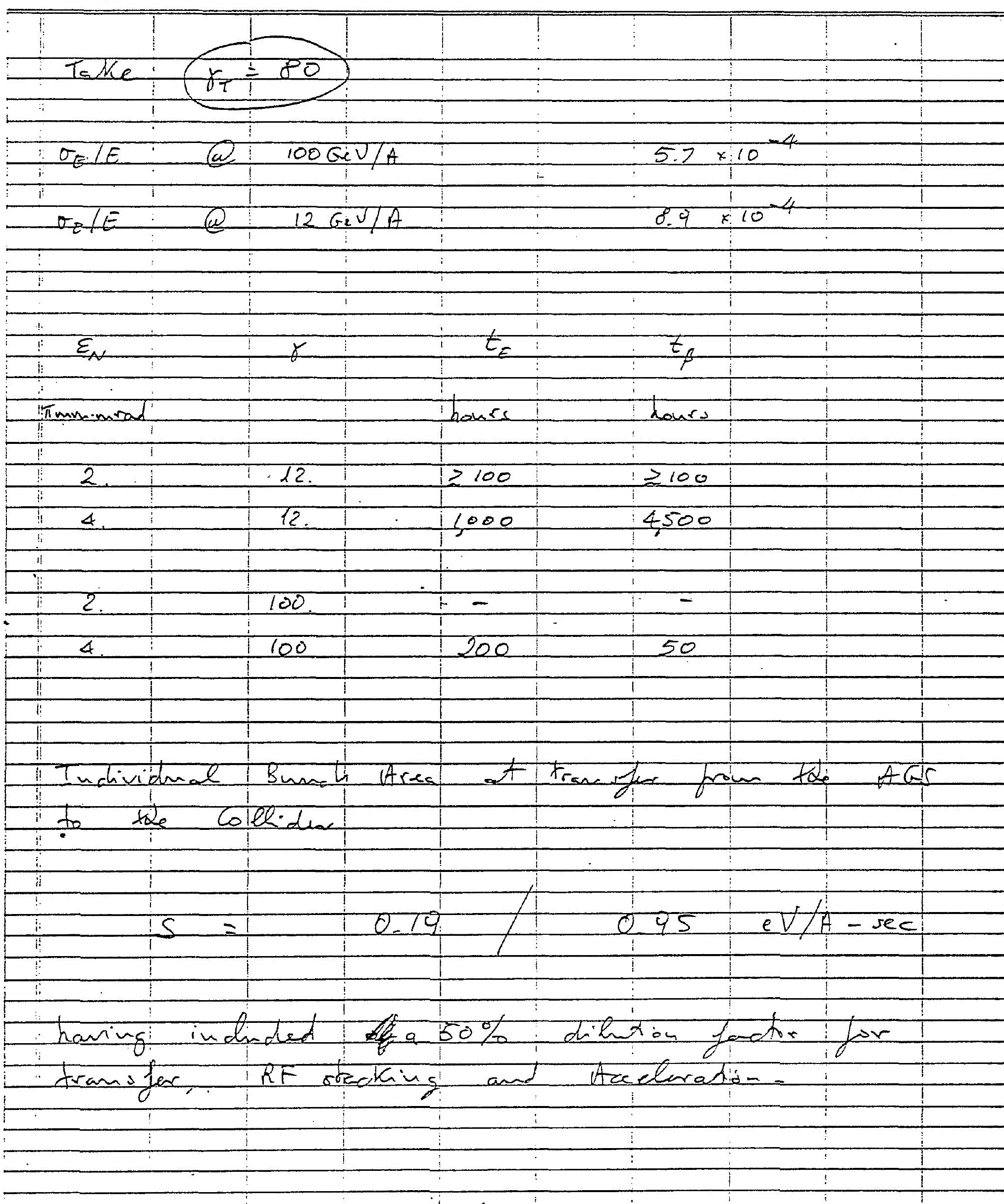
Le lnminasty of $1.8 \times 10^{27} \mathrm{~cm}^{2}$, con be abtained:if

$$
\sigma_{v}=\frac{0.037 \mathrm{~min}}{1}
$$

at doe interaction regiven

This cavrejoods: to a $\beta_{v}^{*}=2 \mathrm{~m}$ mith a

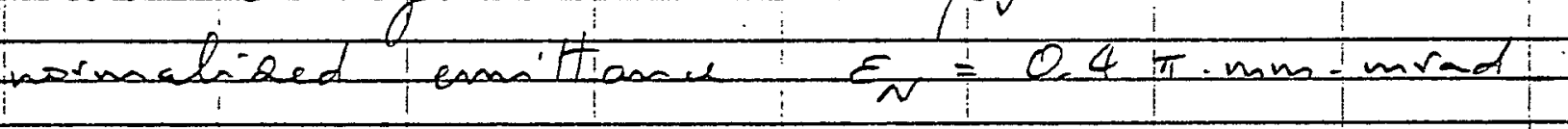

$$
\begin{aligned}
& \text { We: hase wow } \\
& \varepsilon_{N} \\
& \frac{3}{1} \\
& 0.4 \pi \cdot \operatorname{mm} m \text { mad } \\
& 2.0 \\
& 4.0 \\
& \text {.or wixh } \beta_{v}^{*}=2 \text { i } \\
& \varepsilon \\
& 0.4 \pi \cdot m \text { man: } \\
& 20 \\
& 4.0 \\
& \beta_{v}^{*} \\
& 2 m \\
& 0.4 m \\
& 0.2 m \\
& 1 \\
& 1.8 \times 10^{27} \mathrm{~cm}^{2} \mathrm{~s}^{-1} \\
& 0.8 \\
& 0.57
\end{aligned}
$$

